\title{
Machine Learning Techniques for Anxiety Disorder
}

\author{
Elif Altintas ${ }^{1, *}$, Zeyneb Uylas Aksu ${ }^{2}$, Zeynep Gumus ${ }^{3}$ \\ 1* Haliç University, Faculty of Engineering, Department of Software Engineering, Istanbul, 34445, Turkey,, (ORCID: 0000-0002-3454-0326), \\ elifaltintaskahriman@halic.edu.tr \\ 2 Istanbul Bilgi University, School of Advanced Vocational Studies, Department of Computer Technology, Istanbul, 34060, Turkey, (ORCID: 0000-0001-9910-2696), \\ zeynep.aksu@bilgi.edu.tr \\ 3 Uskudar University, Faculty of Humanities and Social Sciences, Department of Psychology, Istanbul, 34662, Turkey, (ORCID: 0000-0003-4474-4813), \\ zeynep.gumus@uskudar.edu.tr
}

(First received 23 September and in final form 23 December 2021)

(DOI: 10.31590 /ejosat.999914)

\begin{abstract}
ATIF/REFERENCE: Altintas, E., Uylas Aksu, Z., \& Gumus Z. (2021). Machine Learning Techniques in the Treatment of Anxiety Disorder. European Journal of Science and Technology, (31), 365-374.

Abstract

In recent years, artificial intelligence based applications have been improved and used to improve the timing, sensitivity and quality of diagnosis of psychiatric diseases. We aim to review the existing literature on the use of artificial intelligence techniques in the assessment of subjects with anxiety disorder. We searched databases about DSM-5 (Diagnostic and Statistical Manual of Mental Disorders) one of the main categories of anxiety disorders; Separation Anxiety Disorder, Generalized Anxiety Disorder, Panic Disorder and Social Anxiety Disorder between 2015-2021. We identified 30 different techniques on these works. Comparisons have been made with more than one algorithm in the studies. The Random Forest Algorithm has been seen in the most used machine learning method among these algorithms. In addition, the best accuracy performance has been observed in the Random Forest Algorithm. This article critically analyzes these recent research studies on anxiety. Considering the clinical heterogeneity of the data obtained from anxiety patients, we conclude that artificial intelligence techniques can provide important information to clinicians and researchers in areas such as diagnosis, personalized treatment, and prognosis.
\end{abstract}

Keywords: Anxiety disorders, Machine Learning, Panic Disorder, Psychiatric Disorders, Social Anxiety Disorder, Random Forest Classifier.

\section{Anksiyete Bozukluğunda Makine Öğrenmesi Teknikleri}

$\ddot{0} \mathbf{z}$

Son yıllarda psikiyatrik hastalıkların teşhisinin zamanlamasını, duyarlılığını ve kalitesini iyileştirmek için yapay zeka tabanlı uygulamalar geliştirilmekte ve kullanılmaktadır. Anksiyete bozukluğu olan deneklerin değerlendirilmesinde yapay zeka tekniklerinin kullanımına ilişkin mevcut literatürü gözden geçirmeyi amaçlanmaktadır. Anksiyete bozukluklarının ana kategorilerinden biri olan; ayrılık kaygısı bozukluğu, genelleşmiş kaygı bozukluğu, panik bozukluğu ve sosyal kaygı bozukluğu DSM-5 (Ruhsal Bozuklukların Tanısal ve İstatistiksel El Kitabı) ile ilgili 2015-2021 yılları arasındaki veri tabanları araştırılmıştır. Bu çalışmalarda kullanılan 30 farklı teknik belirlenmiştir. Yapılan çalışmalarda birden fazla algoritma ile karşılaştırmalar yapılmıştır. Bu algoritmalar arasında en çok kullanılan makine öğrenmesi yönteminde Rastgele Orman Algoritması görülmüştür. Ayrıca en iyi doğruluk performansı Rastgele Orman Algoritması'nda gözlemlenmiştir. Bu makale, kaygı üzerine yapılan bu son araştırma çalışmalarını eleştirel bir şekilde analiz etmektedir. Anksiyete hastalarından elde edilen verilerin klinik heterojenliği göz önüne alındığında, yapay zeka tekniklerinin tanı, kişiselleştirilmiş tedavi ve prognoz gibi alanlarda klinisyenlere ve araştırmacılara önemli bilgiler sağlayabileceği sonucuna varılmıştır.

Anahtar Kelimeler: Anksiyete Bozukluğu, Makine Öğrenmesi, Panik Bozukluk, Psikiyatrik Bozukluklar, Sosyal Anksiyete Bozukluğu, Rastgele Orman Sinflandırıcilar. 


\section{Introduction}

In recent years, Artificial intelligence (AI) with its vast realworld applications play a significant role in science which are disease diagnosis, face recognition, robotics, digital applications, data mining, industrial applications. Artificial neural networks (ANNs) have been used in many fields such as signal processing, pattern recognition (PR) and forecasting, and have achieved high success. The research of ANNs has become a hot spot in the AI field [1]. AI is rapidly emerging and is expected to affect all aspects of medicine, especially psychiatry. PubMed, Embase, Web of Science, ScienceDirect, Scopus, and Google Scholar search for the term "AI in psychiatry" indicate that publications on this topic have increased exponentially in recent years.

Additionally, psychiatrists have increasingly noticed the need for a basic comprehension of AI. Co-operation between data scientists, physicians, computer\&software engineers is needed to provide proper training, operation, and supervision of AI-based systems\&applications [2]. AI-based systems and applications have brought more novel and effective solutions for the treatment of psychiatric diseases. Recent improvements in technology\&computer science include human-like machines that imitate human behavior or cognitive processing [3].

Since medical diagnosis comprises uncertain highdimensional clinical data, AI technology is urgently needed to deal with the various properties of data, which will further work up and help medical practitioners to make effective and accurate decisions [4]. Diagnosis could been difficult due to the common symptoms of many psychiatric disorders (PDs). With machine learning approaches, similar symptoms of diseases could be detected. Kurban determined the closeness of text-based fields with each other using machine learning methods [47].

Psychological disorder is a sociable issue that can cause severe suffering or harm to human work. At present, mental illness is one of the five main syndromes that cause deficiency to work, constituting more than $30 \%$ of all diseases in a generation. In recent years, new techniques for diagnosis mental illness and its effects, causes and treatment methods have been studied and established by many researchers [5].

In accordance with DSM-5 [6], psychological and psychotic disorders can be divided into neuro developmental, bipolar and relative, depression, anxiety, obsessive-compulsive, mental related to obsessive-compulsive, separation Sexual, eating and eating, elimination, arousal, impulsive, addictive, neurocognitive, personality and relatives and friends sexual disorders [6] .

With the improvement of technology, research on the brain has increased to understand the mechanisms and etiology of PDs $[7,8,9]$. Psychiatric research and applications based on AI for diagnosis, which are thought to help to understand the pathophysiology of mental disorders, are on the agenda of health policies [10]. It is thought that developing technology and AI techniques will be useful in evaluation, diagnosis, treatment and clinical decision making $[11,12]$.

In light of the above, the aim of this study is to systhematically review, turn to account, and compare available methods designed to identify the best performing techniques to foresee anxiety diseases in early stage and which algorithm works best with what type of data.

\section{Material and Method}

More than 500 articles published from 1990 to 2015 have been reviewed and 200 were selected for this survey. These articles are 19 depressive disorders, 63 schizophrenias, 112 $\mathrm{AD} / \mathrm{MCI}, 22 \mathrm{ADHD}$ and $20 \mathrm{ASD}$. In MCI/AD studies due to Alzheimer's Disease Neuroimaging Initiative (ADNI) dataset, structural MRI has been seen as the most popular method. In ADHD and schizophrenia studies, combined rest and task fMRI have been pointed as the most popular method. High accuracy rates were obtained in studies with a sample size less than 100 [13].

\subsection{Studies on ML and DL}

Neuroimaging on the human brain has become increasingly important in recent years with imaging methods which are magnetic resonance imaging (MRI), magnetoencephalography (MEG), electroencephalography (EEG). Neuroimaging offers new approaches to understand diseases for instance schizophrenia, bipolar disorder, autism spectrum disorder (ASD), Alzheimer's disease (AD), major depressive disorders, attention deficit hyperactivity disorder (ADHD) and mild cognitive impairment (MCI). There are two objectives: Firstly, a large number of MRI-based brain disorder diagnostic/prognostic studies in schizophrenia, ASD, ADHD, depressive disorder, MCI and $\mathrm{AD}$ have been examined and compared according to their type of features, classifier and reported accuracies. Secondly, an opinion has been constructed about how machine learning (ML) can be applied to neuroimaging [13].

Anxiety disorders and depression has among the PDs that are common all over the world. The fast pace of the modern world has effective in the spread of these diseases [14]. Fear and anxiety are the main emotions highly related to evolution. As our most basic "alert system", they ensure that organisms survive in potentially harmful and dynamically changing environments. The defense system of organisms is affected by both (epi) genetic factors and environment, including personal learning experience $[15,16]$. Anxiety disorders are characterized by onset in childhood and early adolescence [17]. Worldwide, anxiety disorders constitute an important part of global diseases (14.6\% of disability-adjusted life years [DALYs]) [18]. Panic disorder is an anxiety disorder in which similar symptoms are seen and accompanied by the anxiety of experiencing recurrent panic attacks after at least one panic attack. Anxiety disorders are often seen together with mood disorders [19].

Psychosocial factors, neurobiological and neuropsychological dysfunctions are thought to be effective in the aetiology of anxiety disorders [20]. Within the scope of anxiety disorders to DSM-5 classification; Generalized Anxiety Disorder (GAD), Separation Anxiety Disorder (SAD), Panic Disorder, Social Phobia, Selective Mutism, Agoraphobia, Specific Phobias, Substance / Drug Induced Anxiety Disorder, and Anxiety Disorder related to another health condition [19].

Rapid processing and analysis of relevant data in anxiety diseases is critical. The use of AI models will help identify many PDs at an earlier or prodromal phase. Thus, more effective interventions will be provided [21]. Increasing AI studies in mental health research and clinical care seem to be the changes brought by the modern world and advancing technology.

Biomarker, one of the scanning and pattern methods, has been used in the diagnosis of the brain image. Wolfers et al. [22] 
have examined the use of patient screenings for psychiatric diagnosis diseases in 2015. According to their reviews, they have thought that there might be some difficulties in PR. In this context, it has been emphasized that pattern scanning is of high signficture in the diagnosis of the disease since the day, it was used [22].

ML contributes to the early diagnosis and treatment of many psychiatric diseases. There are many methods of psychiatric diseases. Cornblath et al. [23] have suggested that combining these techniques with ML will create a new approach to the treatment of psychiatric diseases [23].

Garcia-Ceja et al. have researched works by using ML in mental health monitoring systems (MHMS). They have classified the study in three part: study type which is occurred by connection, detection and forecasting, study duration which is separated to short term and long-term, sensing types which are wearable, external and software/social media. In their study, the papers have classified according to these categories. It has been expressed that that the use of multiple sensing technologies together with ML methods provides an advantage in the treatment of mental diseases [24]. Continuous monitoring of different mental states such as depression, anxiety, stress, and mental health with such applications provides superior efficiency and integrates the field of psychiatry with advanced technology.

Trumpff et al. [25] have determined psychological and physiological spesifiers of ccf-mtDNA reactivity using ML. The study has included 46 healthy middle-aged adults who are $28 \mathrm{men}$, 18 women and between 41-59 years old. ML model was trained using serum ccf-mtDNA concentration data measured before and after stress. 56 traits such as psychological variables, gender, personality traits were classified and have been trained for partial least squares (PLS) were trained for differential analysis (PLS$\mathrm{DA}$ ) and random forest (RF) classification models. It is determined that $\% 60$ women, $\% 40$ men among low responders and $\% 21$ women, $\% 79$ men among high responders $(\mathrm{X} 2=26.95 ; \mathrm{p}$ $<0.0001)$. They concluded that the gender is important factor. Compared with low responders, high responders have a more significant reduction in fatigue caused by stress $(d=0.38)$.

There are many studies related to stress. Smets et al. [26] have researched stress measurement in an experimental environment depending on the physiological signal of the responses. They have compared ML techniques in research. Electrocardiogram (ECG) and galvanic skin response (GSR), heat and respiratory of the subjects were measured. The research has been compared with 6 different ML methods: Logistic Regression (LR), Support Vector Machines (SVMs), Decision Trees (DT), RFs, Bayesian Networks (BNs). The best results have $84.6 \%$ for $\mathrm{BNs}, 82.7 \%$ for generalized SVMs.

According to data from Google scholars, the past ten years have been related to anxiety, attention deficit hyperactivity disorder, autism, stress, insomnia, schizophrenia, depression, Parkinson's, AD and the use of data mining to diagnose dementia. However, only $0 \%, 2 \%$ and $3 \%$ jobs were found in Parkinson, insomnia and anxiety, separately. Abstracts of publications for these diseases are described in the Figure 1 [4]. As seen in the rates, the second least studied area is anxiety. However, according to DSM-5 (Diagnostic and Statistical Manual of Mental Disorders), autism, Parkinson's, Alzheimer's, etc. diseases are considered as neurological diseases that occur in the brain rather than psychological diseases. Genetic scientists, neurologists and image processing specialists work on the preliminary diagnosis and treatment methods of these diseases. Diseases such as anxiety, e-ISSN: 2148-2683 depression and stress are based on psychology and are more likely to be predicted by being evaluated and tested from many different angles, and it is thought that if they can be treated early, their prevalence in society can be prevented. For this reason, anxiety disorder was discussed in this study.

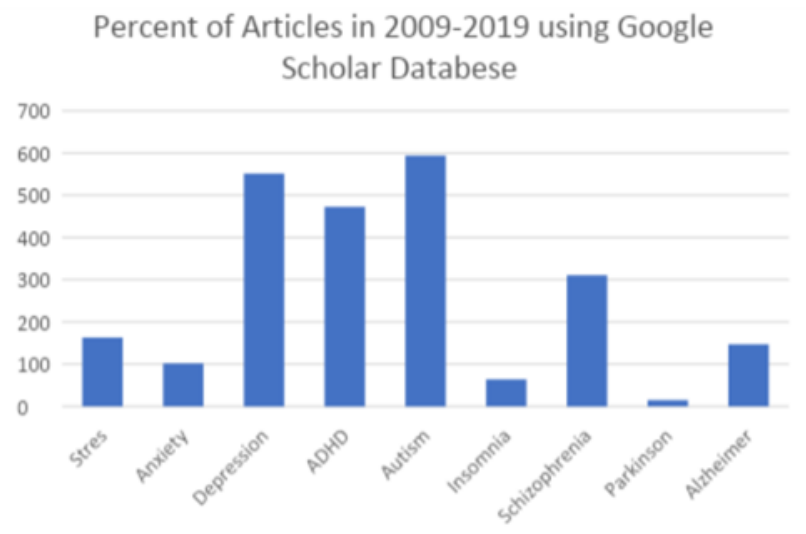

Figure 1. The rate of articles (2009-2019) for diagnosing different psychological disorders.

\subsection{Implementation of ML and DL For Anxiety Disorder}

In the globalizing world, it is difficult to distinguish whether the increasing stress and anxiety in societies living in metropolitan cities is a possible anxiety symptom or periodic emotional states. For this reason, we have compiled studies on anxiety disease, for which early diagnosis is important, and these studies are expected to shed light on future studies in this area.

In 2016, a study prepared by Carpenter et al.[27] has examined anxiety disorder in preschool children. The study includes healthy and sick child profiles aged 2-5 years at pediatric health and diseases clinic of Duke University. "The Preschool Age Psychiatric Assessment" has been applied to children. Two different data groups were used. GAD and SAD have been evaluated separately. The number of participants is 307 in the first data group which has 1073 children, the number of participants is 917 in the second data group which has 3433 children. Test and Education data set has been used. Alternating decision tree (ADTree) which is ML method has been applied. The researchers have compared the j48 algorithm, but J48 has created too many nodes, the comprehensibility has been becoming difficult. While evaluating performance, sensitivity has been measured based on deterministic and accuracy criteria. The algorithm ensure a very reserved risk forecaster for the GAD and SAD, with accuracy values of $97 \%$ and $99 \%$, respectively. It has a sensitivity of $100 \%$, a determination of $97.2 \%$ for GAD and a sensitivity of $99.8 \%$, a sensitivity of $84.7 \%$ for SAD. As a result of the study, the researchers have determined that a short screening tool that defines anxiety risk can be developed, and this risk can be measured in preschool. Here in, it is aimed to reduce the number of factors required to describe a child at risk of worry.

Biomarkers in the field of psychiatry help to predict disease status and outcomes. However, biomarker development is lagging behind in psychiatry due to the heterogeneity in the development of symptoms. Mellem et al. [28] have focused on the classification of psychiatric diseases and conducted a biomarker study based on neuroimaging. Researchers have considered three different models of PDs: dysregulated mood, anhedonia, and anxiety. The data containing the clinical scale evaluations has 
been taken with 272 data from "Consortium for Neuropsychiatric Phenomics". It has included 3 patients with genetic risk, MRI and clinical scale data. Levels of dysregulated mood, anhedonia, and anxiety have been examined. The sample included in the study consists of participants between the ages of 21-50 and this group consists of 4 classes. The healthy individuals $48 \%$, $18 \%$ with schizophrenia, $18 \%$ with bipolar disorder, whereas the remaining 16 percent are those with hyperactivity disorder patients of the total number of participants. Models are constructed with Lasso Regression (L1), Elastic Net Regression, RF. By means of this approach, the model has been optimized, $65 \%$ to $90 \%$ of variance has been explained in 3 symptom areas. According to results, structural features did not play a crucial role in forecasting. Temperament and personality scales have been identified as important predictors of symptom variation. Predictive tolerancestate functional MRI connectivity features have been radiated.

The symptoms of psychiatric diseases are common for many diseases. This situation creates difficulty to diagnose the disease. In a study carried out in 2018, a modeling that predicted anxiety and depression symptoms has been performed with PR [29]. Portugal et al. have examined the shapes of brain images during dynamic emotional facial processing. 74 healthy and 154 young and adult individuals consulting treatment for psychological distress have attended. Individuals who participated in the study and applied for treatment have been DSM-5 diagnoses of depressive disorder, anxiety disorder, eating disorder, exclusionary disorder, trauma related disorder, sleep disorder, stomato-form disorder, and adjustment disorder. The average age of the participants is 21.6 and three quarters of the participants are women. They took to Magnetic Resonance Imaging (MRI) when the participants had fearful, angry and happy faces. They have analyzed to results with Gaussian Process Regression (GPR). A permutation test has been performed to estimate the significance level. As a result of the study, the researchers have emphasized that neuroimaging techniques may be a method for early diagnosis.

Depression could be appeared in many people with panic disorder. It may be necessary to have an idea about the brain waves of panic disorder patients. In this context, Lueken et al. [30] have researched the relationship of fear effect with brain images in individuals with panic disorder. They have taken the brain pictures of 59 panic attack patients with magnetic resonance imaging(MRI). They have examined these pictures with ML methods. They have used the Random Undersampling Boost algorithm (RUSBoost). The study has been resulted in $73 \%$ accuracy, $77 \%$ sensitivity, $70 \%$ specificity. According to the results, it has been observed panic disorder relation during fear in the patients. The researchers have able to explain the presence of external factors when researched the neurofunctional substrates of anxiety disorders. The comorbid can be observed with neurofunctional data. In this context, individualized treatments may also be possible.

Social anxiety disorder is a widespread mental disease that causes depression and panic attacks unless it is treated. Cognitive behavioral therapy (CBT), an effective treatment method, is applied to patients with SAD. However, after long-term treatment has ended, it is difficult to predict the patient's response to treatment. Mannson et al. [31] have researched that 1 year after finishing of Internet-transmitted CBT (iCBT), neural predictors of extended-period cure outcome for twenty six patients. The SVMs have been trained to separate patients who responded and did not respond to treatment according to blood oxygen level-dependent e-ISSN: 2148-2683
(BOLD). There are two models for intervention which are $50 \%$ of the participants onset with $\mathrm{iCBT}$ and the other half onset with ABM. Patients have improved on the Liebowitz Social Anxiety Scale-Self-report version (LSAS-SR) from preprocessing to track. No significant change in disease markers has been detected when evaluated 1 year after treatment. The ratio of longtime responders to treatment has been calculated as $52 \%$ in patients and these results have been obtained with an accuracy of $92 \%$. This analysis has distinguished from patients who respond to treatment from patients who do not respond, and during this analysis, BOLD reaction patterns in the $\mathrm{AACC}$-amygdala regions of the brain have been investigated.

Boeke et al.[32] have used brain images of individuals with anxiety using the data of the Brain Genomics Superstruct Project ( $n=531,307$ women). The model has been constructed by the scikit-learn package in Python. They have used R2 for test phase and they have evaluated various model classes which are ridge regression (L2), L1, partial least squares regression (PLSR), principal components regression (PCR) , Random Forest Regression (RFR), support vector regression (SVR) with a linear or polynomial kernel, relevance vector regression, and the "connectome-based predictive modeling" approach for discovery sample. With the help of permutation test $p$, the best model which is cross-validated R2 of .06 has been found in discovery sample [32]. But unfortunately, they have got failed during the generalization test within the holdout sample with R2 of 2.04, permutation test $\mathrm{p} . .05$.

Considering that there is a relationship between social anxiety and facial feelings in attention processes related to psychopathology, in here focuses on examining this relationship with ML methods. It was done The Eye Movement examination with Hidden Markov Models (HMM) technique. This technique, which can cluster people's eye gestures into dissimilar strategy classes, has been chosen. Eye movements of sixty participants who have mixed level in social anxiety were examined. The eye movements' data for angry faces and neutral faces were start severally in the MATLAB tools. Chan et al. [33] have get the results that people who nonstop used the same strategy on obverse senses were more socially worries than people who altered their tracking strategy among dissimilar obverse senses.

Júnior et al. [34] aim to analyze patterns of rumination disorder from RDoC perspective and to specify which variables predict high levels of incompatible rumination against a trans diagnostic sample by using 200 patients' data with clinical diagnoses of schizophrenia, schizoaffective, bipolar, depression, anxiety disorders, obsessive compulsive and post-traumatic stress. Sociodemographic, serum levels of immune markers and BDNF, psychiatric symptoms and disorders, history of suicide and hospitalizations, functionality, medication use and comorbidities are used for ML algorithm. The prediction accuracy, sensitivity, and specificity of ML in identifying ruminants were determined by a robust cross-validation approach. Greater than possibility accuracy $(>70 \%)$ have get by means of all algorithms.

ML algorithms have been used to determine patient-reported outcome measures (PROMs) which are foretold anxiety disorder and major depressive disorder (MDD) of 637 individuals with immune-mediated inflammatory illness.

PROMS are trained by using neural network (NN), LR and RF models. Tennenhouse et al. [35] get the result that the area below the acceptor manager graph and Brier points in order 
aligned from $0.87-0.91$ for major depressive disorder models and 0.07 for MDD models, and from $0.79-0.83$ and $0.09-0.11$ for anxiety disorder models. NN and LR performed better than RF

Bokma et al. [36] proposed to foresee heal of 887 patients from anxiety disorders. These patients have been applied an ML with RF classifiers within 2 years. At the end of two years 484 patients had recovered. Here $\% 90$ of the data was used for training and $\% 10$ of the data for testing. RF Classifiers realized a crossvalidated area-under-the-receiving-operator-characteristic-curve (AUC) of 0.67. It has aimed to predict the clinical and biological course of anxiety disorders and to predict the state of recovery by using ML technique. By following the individuals for 2 years, moderate predictive performance was observed in the improvement of anxiety disorders. It has been found that the prediction of recovery from anxiety disorders is mainly due to anxiety characteristics; It has been shown that recovery from anxiety disorders and major depressive disorders is mainly due to the characteristics of depression.

To predict SAD, threatening faces can be used. Half of the study group are SAD and half are healthy individuals. Their brain reaction to threatening countenances, threatening countenances, and threatening/happy countenances in 90 areas containing frontal, limbic, parietal, temporal, and occipital systems during functional MRI was used by Xing et al. [37]. Two-tailed independent t-tests and chi-square tests were applied to estimate subscriber typical. SVM model achieved that AUC is 0.72 . Social threat perception has been studied in SAD. Brain regions outside of the fear period have been emerged to be important in predicting SAD [37]. The results have obtained show that activities in the regions that manage sensory and goal-oriented handles can perform a part in the diagnostic of SAD and offer a different approach.

In Priyaa et al.'s study [38], data were get and applied on five different ML algorithms between 348 employed and unemployed individuals by using the depression, anxiety and stress scale questionnaire (DASS21). These algorithms are DT, RF tree, naive bayes (NB), SVM and the K Nearest Neighbour (KNN) in R program. In this data set, the number of training and test set are 70 and 30, respectively. The result of the study is that NB has highest precision but the best model is RF. In NB algorithm, accuracies are $0.733,0.855,0.742$ for anxiety, depression stress, respectively.

Kumar et al. [39] get and applied the data on eight different ML algorithms between individuals by using the depression, anxiety and stress scale questionnaire (DASS42 and DASS21). These algorithms are grouped in classes: probabilistic, nearest neighbor, NN, and tree. These algorithms are also naïve bayes, $\mathrm{BN}, \mathrm{k}$-star, local nearest neighbor, multilayer perceptron (MLP), radial basis function (RBF) network, RF and J48 by using WEKA data mining tool. In DASS42 data set, the number of training and test set are respectively 75 and 25 . The result of the study is that, RBF network has highest accuracy, and also the worst model is J48. In RBF network, accuracies are $\% 97.48, \% 96.02, \% 96.17$ for anxiety, depression stress, respectively for DASS42 dataset. When the same methods were applied in DASS21 dataset, if we want to classify according to diseases; for anxiety the best result and accuracy (\%100) was get in Random Forest Algorithm (RFA), for depression the best result and accuracy (\%96.55) was get in RBF network, for stress the best result and accuracy (\%96.55) was get in MLP.
Praveen et. al. [40] analyzed 840.000 tweets that send by 330 million active social media users and use this data by applying sentimental analysis with the python library Textblob and Latent Dirichlet Allocation (LDA) topic modeling over seven months (April 1 to October 30 ). Here the aim is that to understand common persons perspectives of what causes them stress, anxiety, and trauma because of COVID-19, to diagnose at early stage and to formulate treatment method. According to the results of sentimental analysis, Tweets with negative sentiment, Neutral emotional, positive emotional about COVID crises accounted for $34.4 \%(n=289,091), 60.3 \%(n=506,940$ and $5.23 \%(n=43,969)$, respectively. Second method which is LDA topic modeling, determined ten different topics from the corpus and divided the topics into various categories. The percentage of positive, negative, and neutral tweets for each month are presented.

The predictive performances of traditional LR, basic probabilistic ML methods, and automated ML (Auto-sklearn) by using data from the Netherlands Study of Depression and Anxiety which include participants who are 42.2 years old, $66.5 \%$ women, and $53.6 \%$ had a current mood or anxiety disorder were examined to predict depression and anxiety diagnoses at a 2-, 4-, 6-, and 9year follow up [41]. At 4-, 6-, and 9-year follow up, accuracy values ranged between .73-.78, .71-.77, and .76-.79 for LR, NB classifier, and Auto-sklearn, respectively. However, when it comes to evaluating more complex data sets with individual item scores, Auto-sklearn is even better.

The electronic health record collection of a large number of biometric markers and patient characteristics, which can facilitate the detection of GAD and major depression disorder (MDD) in primary care institutions are constituted from a sample of 4,184 undergraduate students [42]. In the training model, 59 biomedical and demographic characteristics and a set of engineering characteristics from general health surveys were determined. The $70 \%(\mathrm{~N}=2929)$ and $30 \%(\mathrm{~N}=1255)$ of data was used for training and testing, respectively. According to XGBoost, RF, SVM, KNN and a NN tuned using Bayesian hyperparameter optimization, data was analyzed. Nemesure et. al. found AUC of 0.73 (sensitivity: 0.66, specificity: 0.7) and 0.67 (sensitivity: 0.55, specificity: 0.7) for GAD, and MDD, respectively.

Researchers have aimed to make dissimilar panic disorder from other anxiety disorders using a ML-based approach with heart rate variability (HRV) between a total of 60 (panic disorder) and 61 patients (other anxiety disorders), (between 20-65 years old) [43]. The used algorithms are RF, gradient boosting machine (GBM), SVM, ANN, and regularized LR. As a result, the best accuracy (0.784), followed by ANN (0.730), SVM (0.730), GBM (0.676) with The L1-regularized LR and RF (0.649) are obtained. LR also is of good performance in other measures, such as F1score $(0.790)$, originality $(0.737)$, sensitivity $(0.833)$, and Matthews correlation coefficient (0.572).

This research was constructed with 102 healthy volunteers (Male/Female: 54/48, between 20-70 years old and the data is divided into two independent data sets: training set and test set [44]. The training set consists of 75 samples, and the training set consists of 27 samples. the model was trained by (GLM) and supervised learning (SL). Photoplethysmography (PPG) was evaluated with a custom system, and participants' state anxiety (SA) was assessed by the State-Trait Anxiety Inventory (STAI-Y) test. As a result, $r=0.81 ; p=1.87 \cdot 10-9$ were obtained and this show that PPG is an up and coming tool for emotion recognizing, which is proper for human-computer interaction applications. 
Nicholas et al.[45] have purposed to estimate the alters in symptoms of major depression and GAD during the period from the previous to nine month trace in a passing controlled test of cross-diagnostic digital interposition based on pre-treatment data of participants with one group admission online concerned meaning therapy with feedback from a therapist $(\mathrm{N}=303)$ and the other group receiving information related to stress management and coping $(\mathrm{N}=329)$. The data were obtained by Patient Health Questionnaire-9 and GAD-7 item questionnaire before the intervention and after a 9-month follow-up and processed in $\mathrm{R}$ and ML modeling was performed in python with Sklearn such as L2, RF, GLM, GPR, Extreme Gradient Boosting (XGBoost), KNN and SVM. The basis characteristics could completely foresee changes in depressive symptoms in both treatment groups. It has demonstrated enabling personalized decision making about which people to refer to standalone digital interventions or mixed-stage care.

\section{Results and Discussion}

\subsection{Comparisons of Training Models}

There are three important types of ML Algorithms: SL which is widely used for data with precise mapping between input and output data, Unsupervised Learning (USL) in which the data is not clearly labeled as a different class, that is, there is no label, Reinforcement Learning which covers more areas of AI, allowing machines to interact with their dynamic environment to achieve their targets. All three paradigms are used everywhere to power intelligent applications and are classified as Figure 2 according to their areas of use.

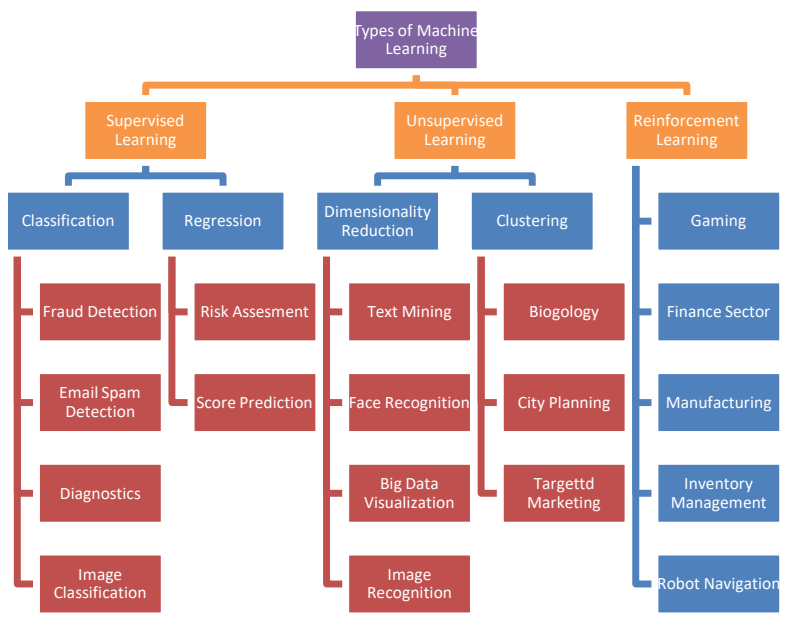

Figure 2. Types of ML algorithms with definite use cases

In this review, original articles between 2015 and 2021 have been investigated. Among these years, two articles in 2015, one article in 2016, 2018, 2019, eight articles in 2020, seven articles in 2021 has examined. In this context, it is possible to say that ML studies on anxiety have increased in recent years.

In the last five years of ML-anxiety studies compiled and has determined that working with 30 different techniques. There have many methods and algorithms in ML which are ADTree, J48, Elastic Net, L1, RFAs, PR, Regression Analysis, RUSBoost, SVM, L2, L1: Principal Component Regression (PCR), RF: Relevance Vector Machine (RVM), PLSR, Linear Or Polynomial Kernel, Critical Path Method (CPM), HMM, NN, LR, NB, KNN, BN, K-Star, RBF Network, Auto-Sklearn, Xgboost, GBM, GLM, e-ISSN: 2148-2683
Wearable sensor technology to foresee longtime (17-18year) deterioration in GAD and panic disorder symptoms from actigraphy data on daytime movement and nighttime sleeping patterns was used for constructing deep learning models which to estimate whether participants experienced increased anxiety disorder symptoms across this period with the participants $(\mathrm{N}=$ 265) [46].

The study has been distinguished to three phase that first phase is occured between 1995-1996 and here in participants carried out telephone interviews to capture mental health symptoms, second between 2004-2009 collecting wearable movement data from participants out of the UW-Madison study site and the last phase is occurred between 2013-2019 by reinterviewing regarding their mental health symptoms. Out-ofsample cross-validated results showed that wearable movement data is important identifier for which people will involvement in worsening of symptoms.

GPR, XGBoost. The selection of these algorithms varies according to the type and amount of data obtained. It has been observed that some algorithms and methods, including Elastic Net, PR, Regression Analysis, PCR, RVM, PLSR, Linear or Polynomial Kernel, CPM, HMM, LR, BN, K-Star, RBF Network, Auto-Sklearn, Xgboost, GBM, GLM, XGBoost have been studied only once. ADTree, GPR, J48, L1, Random Under Sampling, L2, Boost Algorithm, NN, NB, KNN, SVM, RF have took part in more than one study. It has seen that the most used ML method is RFA.

The size of samples in the articles varied. The articles have been completed with a minimum of 26 and a maximum of 330 , 000,000 participants. It has been seen that the number of samples is high for the articles where the RFA gives the best results. In this context, it could be said that the performance value of the RFA is related to the size of samples. Algorithms used in the studies have been compared in the Table 1 in terms of sample size, age, choose modalities, best modality and accuracy performance information. 
Table 1. Comparison of Algorithms used in the studies between 2015 and 2020.

\begin{tabular}{|c|c|c|c|c|c|}
\hline References & $\begin{array}{l}\text { Sampl } \\
\text { e size }\end{array}$ & Age & Techniques & Best Pratice & Performance (\%) \\
\hline$[30]$ & 59 & $18-65$ & RUSBoost & RUSBoost & $73 \%$ \\
\hline$[31]$ & 26 & $19-57$ & SVM & SVM & $92 \%$ \\
\hline [27] & 1224 & $2-5$ & ADTree , J48 & ADTree & $96 \%$ \\
\hline [29] & 154 & $18-25$ & PR, Regression analysis, GPR & GPR & $57 \%$ \\
\hline$[32]$ & 531 & $18-35$ & $\begin{array}{l}\text { L1, RFA, L2, PCR, RVM, PLSR, Linear or Polynomial } \\
\text { Kernel }\end{array}$ & $\begin{array}{l}\text { A stacked } \\
\text { model that } \\
\text { combined } \\
\text { predictions } \\
\text { from } \\
\text { multimodal } \\
\text { data. }\end{array}$ & $95 \%$ \\
\hline [28] & 272 & $21-50$ & Elastic Net, L1, RF & Elastic net & $78 \%$ \\
\hline [33] & 60 & $17-22$ & HMM & HMM & undefined \\
\hline$[34]$ & 200 & $\begin{array}{l}44.14 \pm \\
12.8\end{array}$ & RUSBoost & RUSBoost & $83 \%$ \\
\hline [35] & 637 & 51 mean & RFA, NN, LR & LR & $87 \%$ \\
\hline [36] & 887 & $\begin{array}{l}38.25 \\
\text { mean }\end{array}$ & RFA & RFA & $67 \%$ \\
\hline [37] & 94 & $18-65$ & SVM & SVM & $72 \%$ \\
\hline [38] & 348 & $20-60$ & DT, RFA, NB, SVM, KNN & RFA & $73 \%$ \\
\hline [39] & 39,776 & $18-60$ & NB, BN, k-Star, KNN, MLP, RBF Network, RFA, J48 & RFA & $100 \%$ \\
\hline [40] & $\begin{array}{l}330, \\
000,00 \\
0\end{array}$ & $\begin{array}{l}\text { some } \\
\text { Indian } \\
\text { Twitter } \\
\text { users }\end{array}$ & Textblob Sentiment Algorithm, LDA & LDA & Undefined \\
\hline [41] & 2,981 & 42.2 & LR, NB, Auto-Sklearn & Auto-Sklearn & $79 \%$ \\
\hline [42] & 4,184 & $\begin{array}{l}\text { Less } \\
\text { than } 18, \\
18,19,20 \\
\text { and more } \\
\text { than } 20\end{array}$ & Xgboost, RF, SVM, KNN, NN & $\begin{array}{l}\text { XGBoost } \\
\text { classifier }\end{array}$ & $73 \%$ \\
\hline [43] & 121 & $20-65$ & RFA, GBM ,SVM, ANN & ANN & $78 \%$ \\
\hline [44] & 102 & $20-70$ & GLM and SL & GLM & $81 \%$ \\
\hline [45] & 632 & undefined & L2, RF, GLM, GPR, XGBoost , KNN, And SVM. & GLM & $95 \%$ \\
\hline [46] & 265 & $22-75$ & $\begin{array}{l}\text { A deep auto-encoder paired with a multi-layered } \\
\text { ensemble deep learning model and ensemble pipeline }\end{array}$ & $\begin{array}{l}\text { A deep auto- } \\
\text { encoder paired } \\
\text { with a multi- } \\
\text { layered } \\
\text { ensemble deep } \\
\text { learning } \\
\text { model and } \\
\text { ensemble } \\
\text { pipeline }\end{array}$ & $69 \%$ \\
\hline
\end{tabular}


Among the articles, only one has worked with childhood. The scarcity of the study indicates that useful studies could be made for early diagnosis for these age groups. Other articles have been studied with participants who were in adulthood. It is noteworthy that there is no study on adolescence among the studies. So much so that it could be interpreted that the selection was not preferred because it was difficult to detect anxiety in this period.

\section{Conclusions and Recommendations}

ML technology has been applied to about all areas of human life to help and improve the problem solving ability of the system. With the application of these technologies, the field of psychiatry has improved to a greater extent. Psychiatric diagnosis methods have been implemented using a variety of ML methods, such as SVMs, NNs, Fuzzy Logic, DTs, etc., to help psychiatrists make precise disease diagnosis. Although all these techniques have improved precision, it is important to apply the correct algorithms of these methods with available data. Examined studies have been compared for sample size, age, techniques, best practice, performance values in Table 1 . However, since f-mesure value and other some measure values have not been shared equally in most of the studies, table comparison has been limited. In addition, the tool used in data analysis has be also important in terms of evaluating the results of the study. Lack of this information in most of the studies has been another limitation in terms of comparison. In this review, various researches are examined and analyzed with respect to algorithms of ML. Between these algorithms, RFA is the most preferred because of the best results obtained. RFA works with categorical data instead of numerical data. Data from patients have diagnosed with anxiety disorder are expected to be square. For this reason, it is meaningful to choose more algorithms that work with categorical data. In addition, a theoretical procedure is proposed that can help researchers to understand the complex relationship between human intelligence, body and behavior. These models, which are a new approach in the field of neuropsychiatry, are thought to have a critical role in neuropsychiatric care in the future. The fact that these techniques can process an unlimited amount of data will make a great contribution and guide the clinicians. In some researches which conducted to predict symptom severity of mood, anhedonia, and anxiety, have found that a core panel of symptoms mostly found in several PDs which have a heterogeneous nature, can be predicted. The fact that it can be applied in a shorter time and widely, and that it defines the symptom severity with high anticipation will make a significant contribution to psychiatry. In addition, PR methods that can be of clinical benefit in the evaluation of PDs will provide a comprehensive perspective to clinicians. Prompt diagnosis and treatment in young children with continuing brain development may minimize the risk of psychiatric illness later in life. The increase in reliable, affordable and accessible screening tools for this age group will make it easier to recognize early-onset anxiety disorders. It may provide a more widely applicable screening opportunity for multiple symptom areas and differential diagnosis difficulties of PDs. Studies conducted to predict comorbidity at the individual patient level and neural correlations on the strength of the neurological characteristics of agoraphobia and depression in panic disorder provide data that will contribute to the field with accurate comorbidity predictions. It is thought that study examining changes in eye movements of socially anxious people studies, which offer a new perspective on the relationship between the symptoms of psychopathologies and their relationship, will create an advantage in the diagnosis and treatment processes. The results showed that studies on the pharmacogenetics of anxiety disorders with ML models will guide the discovery of possible functional mechanisms.

\section{References}

1. Yang, X., J. Lin and W. Zheng, Research on learning mechanism designing for equilibrated bipolar spiking neural networks. Artif Intell Rev, 2020. 53: p. 5189-5215. https://doi.org/10.1007/s10462-020-09818-5

2. Górriz, J.M., J. Ramírez, A. Ortíz, F.J. Martínez-Murcia, F. Segovia, J. Suckling, M. Leming, Y.D. Zhang, J.R. ÁlvarezSánchez, G. Bologna, P. Bonomini, F.E. Casado, D. Charte, F. Charte, R. Contreras, A. Cuesta-Infante, R.J. Duro, A. Fernández-Caballero, E. Fernández-Jover, P. Gómez-Vilda, M. Graña, F. Herrera, R. Iglesias, A. Lekova, J.D. Lope, E. López-Rubio, R. Martínez-Tomás, M.A. Molina-Cabello, A.S. Montemayor, P. Novais, D. Palacios-Alonso, J.J. Pantrigo, B.R. Payne, F.P. López, M.A. Pinninghoff, M. Rincón, J. Santos, K. Thurnhofer-Hemsi, A. Tsanas, R. Varela and J.M. Ferrández, Artificial intelligence within the interplay between natural and artificial computation: Advances in data science, trends and applications. Neurocomputing, 2020. 410:p. 237-270, 0925-2312, https://doi.org/10.1016/j.neucom.2020.05.078

3. Tuena, C., M. Chiappini, C. Repetto and G. Riva, Artificial Intelligence in Clinical Psychology. Reference Module in Neuroscience and Biobehavioral Psychology, Elsevier, 2022, ISBN 9780128093245, https://doi.org/10.1016/B978-0-12818697-8.00001-7.

4. Kour, H., J. Manhas and V. Sharma, Usage and implementation of neuro-fuzzy systems for classification and prediction in the diagnosis of different types of medical disorders: a decade review. Artif Intell Rev 2020. 53: p. 4651-4706. https://doi.org/10.1007/s10462-020-09804-X

5. Riaz, M. And M.R. Hashmi, m-polar neutrosophic soft mapping with application to multiple personality disorder and its associated mental disorders. Artif Intell Rev, 2020. https://doi.org/10.1007/s10462-020-09912-8

7. Iritani S, C. Habuchi, H. Sekiguchi and Y. Torii, Brain research and clinical psychiatry: establishment of a psychiatry brain bank in Japan Nagoya J Med Sci, 2018. 80 (3): p. 309-315. 10.18999/nagjms.80.3.309

8. Poo, M.M., J.L. Du, N.Y. Ip, Z.Q. Xiong, B. Xu and T. Tan, China Brain Project: basic neuroscience, brain diseases, and brain-inspired computing Neuron, 2016. 92 (3) : p. 591-596. 10.1016/j.neuron.2016.10.050

9. Rose, N., The Human Brain Project: social and ethical challenges. Neuron, 2014. 82 (6): p. 1212-1215. https://doi.org/10.1016/j.neuron.2014.06.001

10. Liu, G.D., Y.C. Li, W. Zhang and L. Zhang L, A brief review of artificial intelligence applications and algorithms for psychiatric disorders. Engineering, 2019. 6(4): p. 462-467. https://doi.org/10.1016/j.eng.2019.06.008

11. Buch, V.H., I. Ahmed and M. Maruthappu, Artificial intelligence in medicine: current trends and future possibilities. Br J Gen Pract, 2018. 68(668): p. 143-144. https://doi.org/10.3399/bjgp18x695213

12. Luxton, D.D., An introduction to artificial intelligence in behavioral and mental health care. In Artificial intelligence in behavioral and mental health care, Elseiver Academic Press, 2016, p. 1-26. 
13. Arbabshirani, M.R., S. Plis, J. Sui and V.D. Calhoun, Single subject prediction of brain disorders in neuroimaging: Promises and pitfalls. Neuroimage, 2017. 145: p. 137-165. https://dx.doi.org/10.1016\%2Fj.neuroimage.2016.02.079

14. Schoevers, R.A., C.D. van Borkul, F. Lamers, M.N. Servaas, J.A. Bastiaansen, A.T.F. Beekman and H. Riese, Affect fluctuations examined with ecological momentary assessment in patients with current or remitted depression and anxiety disorders. Psychological Medicine, 2020. 1: p. 1-10. 10.1017/S0033291720000689

15. Gottschalk, M.G. and K. Domschke K, Novel developments in genetic and epigenetic mechanisms of anxiety. Current Opinion in Psychiatry, 2016.29 (1): 32-38. https://doi.org/10.1097/yco.0000000000000219

16. Schiele, M.A. and K. Domschke, Epigenetics at the crossroads between genes, environment and resilience in anxiety disorders. Genes, Brain, and Behavior, 2017. 17 : p. 1-15. 10.1111/gbb.12423

17. Beesdo-Baum, K. and S. Knappe, (201) Developmental epidemiology of anxiety disorders. Child and Adolescent Psychiatric Clinics of North America, 2012. 21 (3): p. 457478. https://psycnet.apa.org/doi/10.1016/j.chc.2012.05.001

18. Whiteford, H.A., L. Degenhardt, J. Rehm, A.J. Baxter, A.J. Ferrari, H.E. Erskine and T. Vos, Global Burden of disease attributable to mental and substance use disorders: Findings from the Global Burden of Disease Study 2010. Lancet, 2013. 382 (9904): p. 1575-1586. https://doi.org/10.1016/s01406736(13)61611-6

19. American Psychiatric Association, Diagnostic and statistical manual of mental disorders (5th. Ed.). 2013, Washington, DC: APA.

20. Bandelow, B., S. Michaelis and D. Wedekind, Treatment of anxiety disorders. Dialogues in clinical neuroscience, 2017. 19(2): $\quad$ p. $93-107$. https://dx.doi.org/10.31887\%2FDCNS.2017.19.2\%2Fbband elow

21. Graham, S., C. Depp, E.E. Lee, C. Nebeker, X. Tu, H.C. Kim and D.V. Jeste, Artificial intelligence for mental health and mental illnesses: an overview. Current psychiatry reports, 2019. 21(11): 116. 10.1007/s11920-019-1094-0

22. Wolfers, T., J.K. Buitelaar, C.F. Beckmann, B. Franke and A.F. Marquand, From estimating activation locality to predicting disorder: a review of pattern recognition for neuroimaging-based psychiatric diagnostics. Neuroscience \& Biobehaviora, 2015. 157: p. 328-349. https://doi.org/10.1016/j.neubiorev.2015.08.001

23. Cornblath, E.J., D.M. Lydon-Staley and D.S. Bassett DS, Harnessing networks and machine learning in neuropsychiatric care. Current opinion in neurobiology, 2019. 55: p. 32-39. 10.1016/j.conb.2018.12.010

24. Garcia-Ceja, E., M. Riegler, T. Nordgreen, P. Jakobsen, K.J. Oedegaard and J. Tørresen, Mental health monitoring with multimodal sensing and machine learning: A survey. Pervasive and Mobile Computing, 2018. 51: p. 1-26. https://doi.org/10.1016/j.pmcj.2018.09.003

25. Trumpff, C., A. Marsland, R.P. Sloan, B.A. Kaufman and M. Picard, Predictors of ccf-mtDNA reactivity to acute psychological stress identified using machine learning classifiers: A proof-of-concept . Psychoneuroendocrinology, 2019. 107: p. 82-92. 10.1016/j.psyneuen.2019.05.001

26. . Smets, E., P. Casale, U. Großekathöfer, B. Lamichhane, W. De Raedt, K. Bogaerts and C. Van Hoof, Comparison of machine learning techniques for psychophysiological stress detection. In International Symposium on Pervasive Computing Paradigms for Mental Health, 2015. 604: p. 1322. Springer, Cham. 10.1007/978-3-319-32270-4_2

27. Carpenter, K.L., P. Sprechmann, R. Calderbank, and G.S. Egger, Quantifying risk for anxiety disorders in preschool children: A machine learning approach. PloS one, 2016. 11(11) $\mathrm{e} 0165524$ https://doi.org/10.1371/journal.pone.0165524

28. Mellem, M.S., Y. Liu, H. Gonzalez, M. Kollada and W.J. Martin, Machine learning models identify multimodal measurements highly predictive of transdiagnostic symptom severity for mood, anhedonia, and anxiety. Biological Psychiatry Cogn Neurosci Neuroimaging, 2020. 5(1): p. 5667. https://doi.org/10.1016/j.bpsc.2019.07.007

29. Portugal, L.C., J. Schrouff, R. Stiffler, M. Bertocci, G. Bebko, H. Chase and J. Mourão-Mirandaae, Predicting anxiety from wholebrain activity patterns to emotional faces in young adults: a machine learning approach. NeuroImage: Clinical, 2018. 23:

1018. https://dx.doi.org/10.1016\%2Fj.nicl.2019.101813

30. Lueken, U., B. Straube, Y. Yang, T. Hahn, K. Beesdo-Baum, H.U. Wittchen and B. Pfleiderer, Separating depressive comorbidity from panic disorder: a combined functional magnetic resonance imaging and machine learning approach. Journal of affective disorders, 2015. 184: p. 182192. https://doi.org/10.1016/j.jad.2015.05.052

31. Månsson, K.N., Predicting long-term outcome of Internetdelivered cognitive behavior therapy for social anxiety disorder using fMRI and support vector machine learning. Translational psychiatry, 2015. 5(3): e530. https://dx.doi.org/10.1038\%2Ftp.2015.22

32. Boeke, E.A., A.J. Holmes and E.A. Phelps, Toward Robust Anxiety Biomarkers: A Machine Learning Approach in a Large-Scale Sample. Biological Psychiatry: Cognitive Neuroscience and Neuroimaging, 2019. 5(8): p. 799-807. https://doi.org/10.1016/j.bpsc.2019.05.018

33. Chan, F.H., T.J. Barry, A.B. Chan and J.H. Hsiao, Understanding visual attention to face emotions in social anxiety using hidden Markov models. Cognition and Emotion, 2020. 34(8): p. 1704-1710. https://doi.org/10.1080/02699931.2020.1781599

34. Júnior, É.D.M.S., I.C. Passos, J. Scott, G. Bristot, E. Scotton, L.S.T Mendes and G.A. Salum, Decoding rumination: A machine learning approach to a transdiagnostic sample of outpatients with anxiety, mood and psychotic disorders. Journal of psychiatric research, 2020. 121: p. 207-213. 10.1016/j.jpsychires.2019.12.005

35. Tennenhouse, L.G., R.A. Marrie, C.N. Bernstei and L.M. Lix, Machine-learning models for depression and anxiety in individuals with immune-mediated inflammatory disease. Journal of Psychosomatic Research, 2020. 134:110126. 10.1016/j.jpsychores.2020.110126

36. Bokma, W.A., P. Zhutovsky, E.J. Giltay, R.A. Schoevers, B.W. Penninx, A.L. Van Balkom and G.A. Van Wingen, Predicting the naturalistic course in anxiety disorders using clinical and biological markers: a machine learning approach. Psychological Medicine, 2020. 11: p. 1-11. https://doi.org/10.1017/S0033291720001658

37. Xing, M., J.M. Fitzgerald and H. Klumpp, Classification of Social Anxiety Disorder With Support Vector Machine Analysis Using Neural Correlates of Social Signals of Threat. Frontiers in psychiatry, 2020. 11: 144. https://doi.org/10.3389/fpsyt.2020.00144 
38. Priyaa, A., S. Garga and N.P. Tiggaa, Predicting Anxiety, Depression and Stress in Modern Life using Machine Learning Algorithms. Procedia Computer Science, 2020, p. 1258-1267.

39. Kumar, P., S. Garg and A. Garg, Assessment of Anxiety, Depression and Stress using Machine Learning Models. Procedia Computer Science, 2020. 171: p. 1989-1998. https://doi.org/10.1016/j.procs.2020.04.213

40. S.V.Praveen, RajeshIttamalla, GerardDeepak, Analyzing Indian general public's perspective on anxiety, stress and trauma during Covid-19 -A machine learning study of 840,000 tweets, Diabetes \& Metabolic Syndrome: Clinical Research \& Reviews, Volume 15, Issue 3, May-June 2021, Pages 667-671, https://doi.org/10.1016/j.dsx.2021.03.016 41. Wessel A. van Eeden, Chuan Luo, Albert M. van Hemert, Ingrid V.E. Carlier, Brenda W. Penninx, Klaas J. Wardenaar, Holger Hoos, Erik J. Giltay, Predicting the 9-year course of mood and anxiety disorders with automated machine learning: A comparison between auto-sklearn, naïve Bayes classifier, and traditional logistic regression, Psychiatry Research, Volume 299, 2021, 113823, ISSN 0165-1781, https://doi.org/10.1016/j.psychres.2021.113823

42. Matthew D. Nemesure, Michael V. Heinz, Raphael Huang \& Nicholas C. Jacobson, Predictive modeling of depression and anxiety using electronic health records and a novel machine learning approach with artificial intelligence, Scientific Reports 11, 2021. https://doi.org/10.1038/s41598021-81368-4

43. Kyoung-SaeNaa, Seo-EunChob, Seong-JinChoa, Machine learning-based discrimination of panic disorder from other anxiety disorders, Journal of Affective Disorders, Volume 278, 1 January 2021, 1-4, https://doi.org/10.1016/j.jad.2020.09.027

44. David Perpetuini, Antonio Maria Chiarelli, Daniela Cardone, Chiara Filippini, Sergio Rinella, Simona Massimino, Francesco Bianco, Valentina Bucciarelli, Vincenzo Vinciguerra, Piero Fallica, Vincenzo Perciavalle, Sabina Gallina, Sabrina Conoci, Arcangelo Merla, PeerJ, Prediction of state anxiety by machine learning applied to photoplethysmography data, 2021 Jan 15;9:e10448. doi: 10.7717/peerj.10448. PMID: 33520434; PMCID: PMC7812926

45. Nicholas C.Jacobson, Matthew D.Nemesure, Using Artificial Intelligence to Predict Change in Depression and Anxiety Symptoms in a Digital Intervention: Evidence from a Transdiagnostic Randomized Controlled Trial, Psychiatry Research, Volume 295, 2021, 113618, ISSN 0165-1781, https://doi.org/10.1016/j.psychres.2020.113618

46. Nicholas C. Jacobson, Damien Lekkas, Raphael Huang, Natalie Thomas, Deep learning paired with wearable passive sensing data predicts deterioration in anxiety disorder symptoms across 17-18 years, Journal of Affective Disorders, Volume 282, 2021, Pages 104-111, ISSN 0165-0327, https://doi.org/10.1016/j.jad.2020.12.086

47. Kurban, H. (2021). Metin Madenciliği ile Tibbi Tedavi Alanlarının Yakınlıklarının Ölçülmesi . Avrupa Bilim ve Teknoloji Dergisi , (21), 518-526. 10.31590/ejosat.833199 